\title{
Group velocity and causality in standard relativistic resistive MHD
}

\author{
Shinji Koide ${ }^{1}$ and Ryogo Morino ${ }^{2}$ \\ 1) Department of Physics, Kumamoto University, 2-39-1, Kurokami, Kumamoto, \\ 860-8555, JAPANa) \\ 2) RKK Computer Service Company, 1-5-11, Kuhonji, Kumamoto, 862-0976, \\ $J A P A N$
}

(Dated: 13 November 2018)

Group velocity of electromagnetic waves in plasmas derived by standard relativistic resistive MHD (resistive RMHD) equations is superluminal. If we assume that the group velocity represents the propagation velocity of a signal, we have to worry about the causality problem. That is, some acausal phenomena may be induced, such that information transportation to the absolute past and spontaneous decrease in the entropy. Here, we tried to find the acausal phenomena using standard resistive RMHD numerical simulations in the suggested situation of the acausal phenomena. The calculation results showed that even in such situations no acausal effect happens. The numerical result with respect to the velocity limit of the information transportation is consistent with a linear theory of wave train propagation. Our results assure that we can use these equations without problems of acausal phenomena.

PACS numbers: 03.30+p, 03.65.Pm

\footnotetext{
a) Electronic mail: koidesin@sci.kumamoto-u.ac.jp
}

b)Electronic mail: morino@rkkcs.co.jp 


\section{INTRODUCTION}

Recently, relativistic ideal MHD (ideal RMHD) equations have been used more frequently to perform numerical simulations of plasmas around black holes $\frac{1}{2} 2$. The ideal RMHD numerical simulations showed that around a rapidly rotating black hole, anti-parallel magnetic field configuration, where the magnetic reconnection will be caused spontaneously, is formed naturally. Furthermore, they showed that much electromagnetic energy is transported into the region between the accretion disk and the black hole ergosphere, stored in the region, and then will be released due to the magnetic instability or magnetic reconnection. To perform calculations of the magnetic reconnection around black holes, we have to use the "resistive" RMHD equations, including resistivity, but no one succeeded in such a calculation yet. This is because we worry that "standard" equations of the resistive RMHD with the "standard" relativistic Ohm's law may have the causality problem, and "generalized" RMHD equations ${ }^{6}$, where the group velocity of the electromagnetic wave in the plasma with the plasma parameter larger than unity is smaller than the light speed $c$, are very complex to perform numerical calculations. That is, with respect to the standard equations, we had a concern that the group velocity $v_{\mathrm{g}}$ of the electromagnetic wave becomes larger than the light speed $c$ and it destroys causality if we assume the group velocity means the propagation speed of transportation of information or energy $\mathrm{y}^{\underline{3}}$. For example, it is suggested that in a coordinates frame where the plasma flows with the velocity larger than the critical velocity $c^{2} / v_{\mathrm{g}}$, the amplitude of the wave packet increases spontaneously ${ }^{4}$. This kind of instability contradicts to causality and the second law of the thermodynamics. On the other hand, using a linear theory of electromagnetic wave propagation, we can prove that no signal propagates with a velocity greater than the light speed in a plasma and such acausal phenomenon never happens as discussed by Jackson ${ }^{3}$ (see Appendix VD).

To solve confusion with respect to causality of the standard resistive RMHD equations, we calculated expected situations of such acausal phenomena (instability) using the standard resistive RMHD equations in a flat space-time. In conclusion, the numerical calculations showed that such acausal phenomena never happen. That is, in such cases that acausal phenomena are expected, the edge of the wave packet becomes dominant compared to the bulk part of the wave packet and the edge of the wave propagates within the light speed. This means that superluminal group velocity does not mean breakdown of causality as 
discussed before ${ }^{4}$. That is, the standard resistive RMHD equations permit the superluminal propagation of the infinite wave packet but not superluminal information propagation, thus do not break causality. We can use the standard resistive RMHD equations within causality. It is noted that in a real plasma, group velocity of the electromagnetic wave is equal to or less than the light speed due to the inertia effect and momentum of electrons or electric current, which are neglected in the standard resistive RMHD. To treat these effects, we have to use "generalized" RMHD equations 5,6 .

In this paper, we report numerical calculations of electromagnetic wave packets using standard resistive RMHD equations. In Section III, we introduce the standard resistive RMHD equations and explain the causality problem suggested before. In Section III, we show a numerical simulation of the electromagnetic wave in uniform, rest, resistive plasma, and in Section [V] we try to calculate the propagation of the electromagnetic wave packet in the situation where acausal phenomena are expected. In Section $\mathrm{V}$, with respect to one of the causality problems, we clarify we should use the head velocity as the velocity limit of the electromagnetic wave in plasma, not the group velocity based on the linear theory of propagation of electromagnetic plane wave. In Section VI, we discuss validity of the standard resistive RMHD equations for astrophysical relativistic plasmas. In Section VII, we summarize the results of the numerical calculations and present some remarks.

\section{STANDARD RESISTIVE RMHD AND SUGGESTED CAUSALITY PROBLEMS}

The standard resistive RMHD equations with 4-form in Minkowski spacetime are as follows:

$$
\begin{aligned}
\partial_{\nu}\left(\rho U^{\nu}\right) & =0, \\
\partial_{\nu} T^{\mu \nu} & =0, \\
\partial_{\nu}{ }^{*} F^{\mu \nu} & =0, \\
\partial_{\nu} F^{\mu \nu} & =J^{\mu}, \\
U_{\nu} F^{\mu \nu} & =\eta\left(J^{\mu}-\rho_{\mathrm{e}}^{\prime} U^{\mu}\right),
\end{aligned}
$$

where $\partial_{\nu}, U^{\nu}$, and $J^{\nu}$ are the 4-derivative in Minkowski spacetime, 4-velocity, and 4-current density, $F^{\mu \nu}$ is the field strength tensor, ${ }^{*} F^{\mu \nu}$ is the dual tensor of $F^{\mu \nu}$, and $T^{\mu \nu}$ is the 
Group velocity and causality in standard resistive RMHD

energy-momentum tensor,

$$
T^{\mu \nu} \equiv p g^{\mu \nu}+h U^{\mu} U^{\nu}+F_{\sigma}^{\mu} F^{\nu \sigma}-\frac{1}{4} g^{\mu \nu} F^{\kappa \lambda} F_{\kappa \lambda}
$$

(see Jackson ${ }^{\underline{3}}$, Weinberg ${ }^{\underline{7}}$ ). The scalar variables, density $\rho$, pressure $p$, enthalpy density $h$, charge density $\rho_{\mathrm{e}}^{\prime}=-U_{\nu} J^{\nu}$ are observed by the rest frame of the plasma. Equation (5) presents the standard relativistic Ohm's law. Through this paper, we use units so that the light speed is unity, $c=1$.

The $3+1$ formalism of the standard resistive RMHD equations is written as,

$$
\begin{gathered}
\frac{\partial \gamma \rho}{\partial t}=-\sum_{i} \frac{\partial}{\partial x^{i}} \gamma \rho v^{i} \\
\frac{\partial P^{i}}{\partial t}=-\sum_{j} \frac{\partial}{\partial x^{j}} T^{i j} \\
\frac{\partial \epsilon}{\partial t}=-\sum_{i} \frac{\partial}{\partial x^{i}}\left(P^{i}-\gamma \rho v^{i}\right) \\
\frac{\partial B_{i}}{\partial t}=-\sum_{j, k} \epsilon^{i j k} \frac{\partial}{\partial x^{j}} E_{k}, \\
J^{i}+\frac{\partial E_{i}}{\partial t}=\sum_{j, k} \epsilon^{i j k} \frac{\partial}{\partial x^{j}} B_{k}, \\
\sum_{i} \frac{\partial}{\partial x^{i}} B_{i}=0 \\
\rho_{\mathrm{e}}=\sum_{i} \frac{\partial}{\partial x^{i}} E_{i}, \\
E^{i}+\sum_{j, k} \epsilon_{i j k} v^{j} B_{k}=\frac{\eta}{\gamma}\left(J^{i}-\rho_{\mathrm{e}}^{\prime} U^{i}\right)
\end{gathered}
$$

where $P^{i}=T^{0 i}$ is the 3-momentum density, $\epsilon=T^{00}$ is the energy density, $B^{i}=\frac{1}{2} \sum_{j k} \epsilon^{i j k} F_{j k}$ is the magnetic field, $E^{i}=F^{0 i}$ is the electric field, $\gamma=U^{0}$ is the Lorentz factor, $v^{i}=U^{i} / \gamma$ is the 3-velocity, and $\rho_{\mathrm{e}}=J^{0}$ is the charge density. Using linearized equations of Eqs. (10) (14), we have the dispersion relation of the electromagnetic wave propagating in a uniform, rest plasma:

$$
\omega^{2}-\frac{i}{\eta} \omega-k^{2}=0
$$


where $\omega$ is the angular frequency and $k$ is the wave number of the electromagnetic wave. Here, we assumed the resistivity is constant over the whole plasma. Then, the group velocity of the electromagnetic wave propagating in the uniform plasma is given by

$$
v_{\mathrm{g}}=\frac{2 \eta k}{\sqrt{(2 \eta k)^{2}-1}}>1 .
$$

We found that the group velocity is always larger than the light speed. If we assume that the group velocity is the propagation velocity of information, we can send an information faster than the light speed. That is, this information propagates outside of the causality corn $t= \pm x$ (Fig. 1(a)). Furthermore, when we consider a new inertia coordinates frame $O^{\prime}-x^{\prime} y^{\prime} z^{\prime}$, which moves with the velocity $v_{0}>1 / v_{\mathrm{g}}$ observed by the plasma rest frame $O-x y z$, the signal seems to propagate toward the past $\left(t^{\prime}<0\right.$, but not "absolute" past) (Fig. 1(b)). If we combine this type of information transport in the two streaming plasmas with the relativistic velocity $v_{0}>1 / v_{\mathrm{g}}$, we may be able to send the information to the absolute past. This is strange because if it is true, we can know the today's information (for example, about stock rate) yesterday. This is one type of causality problems discussed before. The other type of causality problem is about spontaneous decrease in the entropy. Fig. 1(a) shows the electromagnetic wave packet propagation with damping. The damping rate is approximately given by $\Im \omega=1 /(2 \eta)$ from Eq. (15), when $k>1 /(2 \eta)$. Here, let me note that the electromagnetic wave and then wave packet do not propagate in the ideal MHD case $(\eta=0)$ because the damping rate is infinite $(\Im \omega \longrightarrow \infty)$. This damping is due to the convergence of electromagnetic energy to thermal energy of plasma. During the damping of the wave packet, the entropy of the system increases. On the other hand, when we see the same wave in the new coordinates frame $O^{\prime}-x^{\prime} y^{\prime} z^{\prime}$ (Fig. 1(b)), we find the electromagnetic wave propagates toward the left direction and grows up. The increased electromagnetic energy of the grown wave packet comes from the thermal energy of the plasma. This process shows the spontaneous decrease in the entropy. This is also a strange phenomenon. If it is true, using this effect, we can solve the energy problem of the world. As shown above, the causality problem is categorized into the following two types:

1. Transport of information toward the absolute past.

2. Spontaneous decrease in the entropy. 
In this paper, we investigate these problems of the standard resistive RMHD using numerical simulations. Basically, we use the method developed by Watanabe et al. $\underline{\underline{8}}$

\section{INFORMATION TRANSPORTATION}

\section{A. Propagation of a simple wave packet}

We consider a simple wave packet propagation with group velocity $v_{\mathrm{g}}$ in a rest, uniform plasma. Here, we take the parameters of the wave packet as,

$$
\sigma=50, \quad k_{0}=2 \pi, \quad \eta=\frac{1}{2^{3 / 2} \pi}, \quad \omega_{0}=\frac{\pi}{\sqrt{2}}( \pm 1-i)= \pm \pi e^{\mp i \pi / 2}
$$

where $\sigma$ is the characteristic length of the wave packet, $k_{0}$ is the typical wave number, and $\omega_{0}$ is the angular frequency. The initial conditions of the wave packet are given by

$$
\begin{aligned}
\rho & =\rho_{0}=1, \\
p & =p_{0}=0.9, \\
\boldsymbol{v} & =\mathbf{0}, \\
E_{x} & =E_{z}=E_{x}^{0}=E_{y}^{0}=0, \\
E_{y} & =E_{y}^{0}=E_{0} \cos 2 \pi x e^{-x^{2} / 2 \sigma^{2}}, \\
B_{x} & =B_{y}=B_{x}^{0}=B_{y}^{0}=0, \\
B_{z} & =B_{z}^{0}=E_{0} \cos \left(2 \pi x+\frac{\pi}{2}\right) e^{-x^{2} / 2 \sigma^{2}}, \\
J_{x} & =J_{y}=J_{x}^{0}=J_{y}^{0}=0, \\
J_{z} & =J_{z}^{0}=2^{3 / 2} \pi E_{0} \cos 2 \pi x e^{-x^{2} / 2 \sigma^{2}} .
\end{aligned}
$$

Through this paper, we set $\sigma=50$ and $E_{0}=0.01$. We show the results of the wave packet propagation in Fig. 2, We clearly found the shift of the wave packet from the initial position with $\Delta x=4.2$ and $\Delta x=7$ at $t=3$ and $t=5$, respectively. This means that the infinite simple wave packet propagates with the group velocity $v_{\mathrm{g}}=1.4$. This confirms the results of Koide ${ }^{\underline{4}}$. It is also shown that the wave packet damps very quickly. (Note that the scales of the vertical axes of the panels in Fig. 2 are quite different.) 


\section{B. Propagation of a wave packet with a head edge}

We consider a more realistic situation of information transport. In this situation, we can not use the infinite length wave packet at least without the head of the wave packet, because it takes infinite time to produce such a wave packet. Figure 3 shows the wave packet propagation which has the edge at the head $x=32, t=0$. The initial condition is given by multiplying the factor

$$
f(x)=\left\{\begin{array}{l}
1 \\
0(x>32)
\end{array}\right.
$$

to each initial perturbation variable of the electromagnetic wave packet expressed by Eq. (17) as,

$$
E_{i}(x)=f(x) E_{i}^{0}(x), B_{i}(x)=f(x) B_{i}^{0}(x) \text {, and } J_{i}(x)=f(x) J_{i}^{0}(x) .
$$

Fig. 3 clearly shows the bulk of the wave packet propagates with the group velocity $v_{\mathrm{g}}=$ 1.4, as shown in the case without the edge of the wave packet (compare the peak of the wave packet part at $x=0$ and $x=4.2$ at $t=0$ and $t=3.0$, respectively). On the other hand, at the front of the wave packet, a relatively smoothed new structure shaped like combined upside-down solitons (we name it "head structure", hereafter) appears and becomes dominant compared with the primary bulk part of the wave packet, because the head structure damps more slowly than the bulk of the packet does with a short characteristic wave length $\lambda_{0}=2 \pi / k_{0}=1$. The front of the head structure propagates with (less than but almost) the light speed. It should be emphasized that the head structure becomes dominant before the bulk part of the wave packet reaches to the initial position of the wave packet front edge. It is noted that even if the main part of the wave packet has an information, we must begin with creating the front of the wave, and then the velocity of the information transport never exceeds the head velocity of the wave, i.e., the light speed. It is clearly shown that the wave packet can't transport the information with the group velocity (which is larger than the light speed in the present case), and then the group velocity is not regarded as the velocity of the information propagation. As shown in Section $\mathrm{V}$, the velocity limit of the information propagation is given by a variable called the head velocity. Now, the first type of the causality problem of the standard resistive RMHD equations, about the information transportation to the absolute past, is solved. 


\section{SPONTANEOUS DECREASE IN ENTROPY OF WAVE PACKET}

We consider the second type of the causality problem in the standard resistive RMHD, that is, the suggested strange process of spontaneous decrease in the entropy of the electromagnetic wave packet. Before we calculate the wave packet propagation in the new coordinates frame $O^{\prime}-x^{\prime} y^{\prime} z^{\prime}$, where the electromagnetic energy of the wave packet is suggested to increase spontaneously, we have to consider the initial condition of the wave packet. When we use the approximation of the very narrow wave-number spectrum of the wave packet ${ }^{4}$, the perturbation variables of the wave packet, for example, the transverse component of the electric field, $E_{y}$ is given by

$$
E_{y}(x, t) \propto \frac{1}{\sqrt{1+i D t / \sigma^{2}}} \exp \left[-\frac{1}{2 \sigma^{2}} \frac{\left(x-v_{\mathrm{g}} t\right)^{2}}{1+i D t / \sigma^{2}}\right] e^{-i k_{0} x-i \omega_{0} t}
$$

where $\omega_{0}=\omega\left(k_{0}\right), v_{\mathrm{g}}=(\partial \omega / \partial k)_{k=k_{0}}$, and $D=\left(\partial^{2} \omega / \partial k^{2}\right)_{k=k_{0}}$. As shown in Appendix A. the profile of the initial condition $\left(\mathrm{Eq} .(\underline{\mathrm{A} 8})\right.$ ) in the new coordinates frame $O^{\prime}-x^{\prime} y^{\prime} z^{\prime}$ monotonically decreases with respect to the coordinate $x^{\prime}$. This is because the point at $x^{\prime}<0, t^{\prime}=0$ corresponds to the past of the original coordinates frame, $t<0$. In the past, the amplitude of the wave packet is (much) larger than the initial profile in the plasma rest frame. This electromagnetic wave with the monotonically decreasing profile in the frame $O^{\prime}-x^{\prime} y^{\prime} z^{\prime}$ cannot be used as the transporter of information. This is because, in the limit of $x^{\prime} \longrightarrow-\infty$, the amplitude of $E_{y}^{\prime}$ observed in the frame $O^{\prime}-x^{\prime} y^{\prime} z^{\prime}$ is approximately given by $\left|E_{y}^{\prime}\right| \sim \exp \left[-\left(\gamma_{0}^{3} v_{0}^{3} D^{2} /\left(\eta \sigma^{2}\right)\right) x^{\prime}\right]$, from Eq. (A14), which is significantly large over an infinite region. Here, $v_{0}$ is the relative velocity of the frame $O^{\prime}-x^{\prime} y^{\prime} z^{\prime}$ and the frame $O-x y z$ and $\gamma_{0}=\left(1-v_{0}^{2}\right)^{-1 / 2}$ (see Eqs. (A5) and (A6)). We cannot create such electromagnetic wave from a start source point of information in finite time and with finite energy. Then, to perform a simulation of the propagation of the signal with the wave packet, we have to introduce an edge at the rear of the wave packet.

To avoid the Lorentz transformation at $-x^{\prime} \gg 1$ of the new coordinates frame, we shift the wave packet with $\Delta x=32$ toward the left side at $t=0$ (Fig. 44). We also confirmed the primary wave packet propagates with the group velocity $v_{\mathrm{g}}=1.4$. We set the edge of the wave packet at the region $-6 \leq x \leq 0$, which is the relaxation zone of the step function at the wave packet edge.

The initial condition is given by multiplying the following factor to each initial variable 
of the electromagnetic wave packet as shown in Eq. (19);

$$
f(x)=\left\{\begin{array}{cl}
0 & (x \leq-l) \\
1-\left(\frac{x}{l}\right)^{2} e^{\left(x^{2}-l^{2}\right) / 2 \sigma^{2}} & (-l<0<0) \\
1 & (x \geq 0)
\end{array}\right.
$$

where $l=6$ through this paper. As the same of the front edge of the wave packet edge, the relatively smoothed structure appears around the wave packet rear edge and becomes dominant compared to the bulk wave packet (Fig. 5(a)). Fig. 5] (b) zooms up the edge of the wave packet and shows that the left edge propagates with the light speed. In this case, we can set the initial condition of the electromagnetic wave as shown in the first panel of Fig. 6. Because the left of the $x^{\prime}$-axis of the new coordinates frame corresponds to the future of the primary coordinates frame $O^{\prime}-x^{\prime} y^{\prime} z^{\prime}$, the bulk part of the wave becomes too small to see. Only the rear edge part of the wave can be seen. The edge part propagates toward the left direction with the light speed and damps (Fig. 6). This is reasonable because the edge part propagates within the light speed in the plasma rest frame (Fig. 5). Then, the second type of expected strange phenomenon of the causality break-down never happens.

\section{WAVE HEAD VELOCITY AS VELOCITY LIMIT OF INFORMATION PROPAGATION}

In addition to numerical results with the electromagnetic wave packet, we theoretically confirm that the group velocity does not give the limit of the propagation velocities of information and energy in this section. With respect to the information velocity, we have confirmed numerically in Section III that the group velocity presents the propagation veloc-

ity of the wave packet without front or rear edge whose Fourier component is a Gaussian function with a narrow spectrum. While most part of the initial profile of the wave packet is concentrated in the region with the length of several $\sigma$, the amplitude of the profile is finite over the whole range, $-\infty<x<\infty$. In the inertia frame, whose relative velocity to the plasma rest frame exceeds the inverse of the group velocity $\left(v_{0}>1 / v_{\mathrm{g}}\right)$, the wave packet does not transport an information because its shape becomes a monotonic function and has no peak (Appendix $\mathrm{A}$ ). Then, we recognize such a wave packet which spread over the whole region $-\infty<x<\infty$ is not accepted as an object of information transporter in relativity. Concerning the energy propagation velocity, in the first place, we note that we cannot define 
the velocity of energy propagation because energy is a temporal component of 4-momentum and we cannot consider the transportation of energy only. In relativity, we have to consider transportation of 4-momentum, not only the energy component. In fact, the group velocity $v_{\mathrm{g}}=\partial \omega / \partial k$ is not a scalar, much less 4 -vector, and then the superluminal group velocity $v_{\mathrm{g}}>c$ is not an invariant statement in any inertial coordinates frame. This contradicts to relativity. Then, we understand the propagation velocity of energy has no physical meaning in relativity.

Instead of the propagation velocity of the wave packet without the edge (i.e., the group velocity), we can introduce the "head velocity",

$$
v_{\mathrm{h}} \equiv \lim _{\omega \longrightarrow \infty} \frac{\omega}{k}
$$

as the velocity limit of the propagation of non-zero fields. The head velocity $v_{\mathrm{h}}$ is a scalar if it is the light speed in a certain coordinates frame, and it is less than the light speed in any inertia frame if it is in a certain frame (Appendix B). It has been already shown that this head velocity represents the information velocity limit in the dielectrics (see section 7.11 of Jackson $\underline{3}$, and references therein).

Here, we prove that the head velocity of the wave provides the velocity limit of information propagation also in a plasma. This proof confirms the results of Section III with respect to the first problem of causality shown in Section III. To investigate the propagation speed of the electromagnetic plane wave front, we consider an injection of the electromagnetic plane wave normally incident from the vacuum on the semi-infinite uniform plasma filling in the region $x>0$, whose boundary locates at $x=0$ at $t=0$ (Fig. 7). We assume the plasma has uniform resistivity $\eta$, and the dispersion relation of the electromagnetic wave is presented by Eq. (15). The index of refraction $n(\omega)$ is given by

$$
n(\omega)=\frac{k(\omega)}{\omega}=\sqrt{1+\frac{i}{\eta \omega}} .
$$

According to Eqs. (7.124) and (7.125) of Jackson ${ }^{3}$, the amplitude of the electric field of the plane wave for $x>0$ is given by

$$
u(x, t)=\int_{-\infty}^{\infty} \frac{2}{1+n(\omega)} A(\omega) e^{i k(\omega) x-i \omega t} d \omega,
$$

where

$$
A(\omega)=\frac{1}{2 \pi} \int_{-\infty}^{\infty} u_{\mathrm{i}}(0, t) e^{i \omega t} d t
$$


is the Fourier transform of the real incident electric field $u_{\mathrm{i}}(x, t)$. Suppose that the incident wave has a well-defined front edge that reaches $x=0$ at $t=0$. Then, the incident electric field $u_{\mathrm{i}}(x, t)$ evaluated just outside the plasma at $x=0^{-}$has the condition,

$$
u_{\mathrm{i}}(0, t)=0 \quad(t<0)
$$

This condition, together with certain mathematical requirements that are "physically reasonable", is both necessary and sufficient to assure that $A(\omega)$ is analytic in the upper half of the complex $\omega$ plane. In this situation, the "physically reasonable" requirement is that $u_{\mathrm{i}}(0, t) \longrightarrow 0$ as $t \longrightarrow \infty$. This is true at least for the wave packet, while for semi-infinite continuous wave train at $0<t<\infty$, the physical reasonable condition is not satisfied 1 . For an example, we consider a simple case of

$$
u_{\mathrm{i}}(0, t)=\left\{\begin{array}{cc}
E_{0} e^{-\nu t} \cos \omega_{0} t & (t \geq 0) \\
0 & (t<0)
\end{array},\right.
$$

where $E_{0}, \nu$, and $\omega_{0}$ are the positive variables (when $\nu=0$, it is not the case of "physically reasonable"). Then, we have

$$
A(\omega)=\frac{i E_{0}}{2 \pi} \frac{\omega+i \nu}{(\omega+i \nu)^{2}-\omega_{0}^{2}} .
$$

It has singular points at $\omega= \pm \omega_{0}-i \nu$ and a zero point at $\omega=-i \nu$ (Fig. 8). It clearly shows that there is no singular point in the upper half of the $\omega$ plane. In the limit of $|\omega| \longrightarrow \infty$, we have $A \longrightarrow \frac{i E_{0}}{2 \pi \omega}$, then $|A| \leq \frac{E_{0}}{2 \pi|\omega|}$. This inequality can be generalized for an arbitrary incident dumping wave train like a wave packet, if we take the constant $E_{0}$ large enough.

We formally evaluate the amplitude presented by Eq. (24) by contour integration. For a plasma case, the path of the contour integral includes the branch point of $k(\omega)=\omega\left(\omega+\frac{i}{\eta}\right)$ at $\omega=0$. To avoid the multi-variable definition of $k(\omega)$ around $\omega=0$, we have to modify the integral path near the branch point $\omega=0$ so as not to cross the branch line between $\omega=0$ and $\omega=-\frac{i}{\eta}$ as shown in Fig 9. Here, the radius of the half circle $C_{\epsilon}$ around $\omega=0$ is infinitesimally small $(\epsilon \longrightarrow 0)$. With the definition of the head velocity $v_{\mathrm{h}}(\mathrm{Eq} .(22))$, the argument of the exponent in Eq. (24) becomes $i \omega\left(x-v_{\mathrm{h}} t\right)$ for large $\omega$, and the closing of the contour can be done in the upper half plane for $x>v_{\mathrm{h}} t$, and the lower half plane for

\footnotetext{
${ }^{1}$ It means that the initial condition of the rear part of the wave very far form the front edge determines the propagation of the wave front. This case is out of our scope.
} 
$x<v_{\mathrm{h}} t$. With respect to the case of $x>v_{\mathrm{h}} t$, this is because we have the inequality with respect to the integration along the large upper half circle $C_{R}: z=\operatorname{Re}^{i \theta}(0 \leq \theta \leq \pi)$, where $R$ is the radius of the half circle $C_{R}$,

$$
\left|\int_{C_{R}} \frac{2}{1+n(\omega)} A(\omega) e^{i k(\omega) x-i \omega t} d \omega\right| \leq E_{0} \frac{\left|1-e^{-\left(x-v_{\mathrm{h}} t\right) R}\right|}{\left(x-v_{\mathrm{h}} t\right) R}
$$

and then the integral along $C_{R}$ vanishes in the limit of $R \longrightarrow \infty$. In Fig. 9, we can see the integration contour consists of $C_{\epsilon}, C_{R}$, and $I_{\epsilon, R}$ for the case of $x>v_{\mathrm{h}} t$. With the analytic nature of $n(\omega)$ and $A(\omega)$ in the upper half of the $\omega$ Riemann plain, the whole integrand in the right-hand side of Eq. (24) is analytic there, and the Cauchy's theorem guarantees that the integral vanishes. We have thus proved that

$$
u(x, t)=0 \quad\left(x>v_{\mathrm{h}} t\right)
$$

provided that $A(\omega)$ and $n(\omega)$ are analytic for $\Im \omega>0$ and $n(\omega) \longrightarrow 1 / v_{\mathrm{h}}$ for $|\omega| \longrightarrow \infty$. Equation (30), together with Eq. (26), establishes that no signal propagates with a velocity greater than the head velocity, in the plasma. This confirms the results of Section III about the velocity limit of the electromagnetic wave packet, but not those of Section IV with respect to the causality problem of decrease in the entropy. In the plasma, the head velocity becomes the light velocity, $v_{\mathrm{h}}=1$.

\section{USAGE OF STANDARD RESISTIVE RMHD EQUATIONS}

Now, we understand the standard resistive RMHD equations have no causality problem, although the group velocity of the electromagnetic wave calculated by these equations exceeds the light speed. We showed that the head velocity of the wave determines the information propagation velocity limit as shown in the previous section. Then, we are free from the restraint of the superluminal group velocity in the standard resistive RMHD equations. Here, we consider the validity and limit of the standard resistive RMHD equations based on a more general point of view using the generalized RMHD equations. We already evaluated each term of the generalized general relativistic MHD (GRMHD) for the plasmas around several astrophysical black holes $\underline{6}$, where we compare these terms with the $\boldsymbol{V} \times \boldsymbol{B}$ term. We compare the terms beyond the standard resistive RMHD equations with the resistive terms 
in generalized relativistic Ohm's law, first,

$$
\begin{aligned}
\frac{1}{n e} \frac{\partial}{\partial t}\left(\frac{\mu h}{n e} \boldsymbol{J}^{\dagger}\right)= & -\frac{1}{n e} \nabla \cdot \boldsymbol{K}+\frac{1}{2 n e} \nabla(\Delta \mu p-\Delta p) \\
& +\left(\gamma-\frac{\Delta \mu \rho_{\mathrm{e}}}{n e}\right) \boldsymbol{E}+\left(\boldsymbol{U}-\frac{\Delta \mu}{n e} \boldsymbol{J}\right) \times \boldsymbol{B}-\eta\left[\boldsymbol{J}-\rho_{\mathrm{e}}^{\prime}(1+\Theta) \boldsymbol{U}\right],
\end{aligned}
$$

in flat space-time, which comes from Eq. (63) of Koide ${ }^{6}$. Here, $n$ is the particle number density $n \equiv \rho / m, \frac{\mu h}{n e} \boldsymbol{J}^{\dagger}$ is the momentum density of the electric current, $\boldsymbol{K}$ is the stress tensor of charge and current, $m$ is the characteristic mass of a particle $m \equiv m_{+}+m_{-}$, $\mu$ is the normalized reduced mass, $\mu=m_{+} m_{-} /\left(m_{+}+m_{-}\right)^{2}, \Delta \mu$ is the normalized mass difference $\Delta \mu=\left(m_{+}-m_{-}\right) /\left(m_{+}+m_{-}\right), \Delta p=p_{+}-p_{-}$, and $\Theta$ is the thermal energy exchange rate from the negatively charged fluid and the positively fluid, where $m_{ \pm}$is the mass of positively/negatively charged particle, $p_{ \pm}$is the pressure of positively/negatively fluid in two-fluid model. The left-hand side of Eq. (31) presents the inertia of current, the first term of the right-hand side shows the transport of electric current momentum, the second term indicates the thermoelectromotive force, and the term $\eta \rho_{\mathrm{e}}^{\prime} \Theta \boldsymbol{U}$ in the last term expresses the redistribution of the thermal energy due to the friction between the two fluids.

We evaluate these terms beyond the standard relativistic Ohm's law except for the last term about thermal energy redistribution of friction.

- Inertia of electric current: the left-hand side of Eq. (31),

$$
\frac{(\text { inertia of current) }}{(\text { resistive term) }}=\frac{1}{\eta J} \frac{1}{n e} \frac{\partial}{\partial t}\left(\frac{\mu h}{n e} J\right) \sim \frac{1}{\eta J} \frac{\omega \mu h J}{(n e)^{2}}=\mu \frac{\omega}{\nu_{\mathrm{e} *}},
$$

where $\omega$ is a characteristic frequency of phenomena, $J$ is a characteristic value of the current density, and $\nu_{\mathrm{e} *}$ is the Coulomb collision frequency between electron and positively charged particle $\left(*=\mathrm{i}\right.$ : ion, ${ }^{*}=\mathrm{e}$ : positron). Here, we used the relations, $\eta=m \nu_{\mathrm{e} *} /\left(n e^{2}\right) \Rightarrow h \nu_{e *} /(n e)^{2}, \omega_{\mathrm{p}}=n e / \sqrt{\mu h}$, and $\omega_{\mathrm{c}}=e n B / h$. The Coulomb collision frequencies of electron-positron $\left(\nu_{\mathrm{ee}}\right)$ and electron-ion $\left(\nu_{\mathrm{ei}}\right)$ are evaluated by

$$
\begin{aligned}
& \nu_{\mathrm{ee}}=1.6 \times 10^{10}\left(\frac{T_{\mathrm{e}}}{e}\right)^{-3 / 2}\left(\frac{n_{\mathrm{e}}}{10^{20}}\right)\left[\mathrm{s}^{-1}\right], \\
& \nu_{\mathrm{ei}}=6.3 \times 10^{9}\left(\frac{T_{\mathrm{e}}}{e}\right)^{-3 / 2}\left(\frac{n_{\mathrm{e}}}{10^{20}}\right)\left[\mathrm{s}^{-1}\right]
\end{aligned}
$$

where unit of $T_{\mathrm{e}} / e$ is $\mathrm{eV}$ and unit of $n_{\mathrm{e}}$ is $\mathrm{m}^{-3}$ (Appendix in Miyamoto ${ }^{9}$ ). Then, these collision frequency are comparable, $\nu_{\mathrm{ee}} \sim 2.5 \nu_{\mathrm{ei}}$. 
Group velocity and causality in standard resistive RMHD

- Thermoelectromotive force: the second term of right-hand side of Eq. (31),

$$
\begin{aligned}
\frac{(\text { thermoelectromotive force })}{(\text { resistive term })}= & \frac{1}{\eta J} \frac{1}{2 n e}|\nabla(\Delta \mu p-\Delta p)| \\
& \sim \frac{1}{\eta J} \frac{1}{2 n e} \frac{1}{L}|\Delta \mu p-\Delta p| \sim \frac{\Delta \mu p-\Delta p}{2 h} \frac{\mu \omega_{\mathrm{p}}^{2}}{\nu_{\mathrm{e} *} \omega_{\mathrm{c}}},
\end{aligned}
$$

where $L$ is a characteristic length of the phenomena.

- Hall effect: the third term of right-hand side of Eq. (31),

$$
\frac{(\text { Hall term })}{\text { (resistive term) }}=\frac{1}{\eta J} \frac{\Delta \mu}{n e}|\boldsymbol{J} \times \boldsymbol{B}| \sim \frac{1}{\eta J} \frac{\Delta \mu}{n e} J B=\Delta \mu \frac{\omega_{\mathrm{c}}}{\nu_{\mathrm{e} *}},
$$

where $B$ is a characteristic value of magnetic field.

In the generalized RMHD equations, the stress tensor is given by

$$
T^{i j}=p \delta^{i j}+h U^{i} U^{j}+\frac{2 \mu \Delta h}{n e}\left(U^{i} J^{j}+J^{i} U^{j}\right)+\frac{\mu h^{\ddagger}}{(n e)^{2}} J^{i} J^{j}+\left(\frac{B^{2}}{2}+\frac{E^{2}}{2}\right) \delta^{i j}-B_{i} B_{j}-E_{i} E_{j} .
$$

The momentum stress tensor of electric current is shown by the third and forth terms of the right-hand side of Eq. (37). The terms are evaluated by comparison with the kinetic stress tensor $h U^{i} U^{j}$ as

$$
\frac{\text { (current momentum tensor) }}{\text { (hydrodynamic stress tensor) }} \sim \frac{2 \mu \Delta h U J}{U^{2} n e h} \leq 2 \mu \frac{J}{U n e} \sim 2 \mu \frac{\eta}{U L} \frac{B}{\eta n e}=\frac{2 \omega \omega_{\mathrm{c}}}{\omega_{p}^{2}} .
$$

The magnetic Reynolds number $S_{\mathrm{M}}$ is defined by

$$
S_{\mathrm{M}}=\frac{U L}{\eta}=\frac{(n e)^{2} U L}{h \nu_{\mathrm{e} *}}=\frac{\mu \omega_{\mathrm{p}}^{2} U L}{\nu_{\mathrm{e} *}} \leq \frac{\mu \omega_{\mathrm{p}}^{2} L}{\nu_{\mathrm{e} *}}=\mu \frac{\omega_{\mathrm{p}}^{2}}{\nu_{\mathrm{e} *} \omega}
$$

where $U$ is a characteristic values of 4 -velocity $|\boldsymbol{U}|$. Table 1 shows the values of these ratios for plasmas around several types of astrophysical black holes with the mass $M_{\mathrm{BH}}$. Here, we assume that the plasma is electron-ion plasmas. We use the wavelength of the fastest-growing magnetorotational instability $(\mathrm{MRI}), L_{\mathrm{CS}}$, which causes the current sheet in the plasma disk as the characteristic length of phenomena around the black holes $L$. It is noted that the wavelength of the fastest-growing mode gives the maximum thickness of the current. Because in the linear stage, the magnetic field lines are bent with the scale of the wavelength and the current sheet is formed with the scale. In the nonlinear stage, the current sheet is pinched until the magnetohydrodynamic equilibrium between the pressure gradient and Lorentz force is achieved. It was shown in numerical MHD calculations (for 
examples, $\underline{10} \underline{\underline{11}})$. The wavelength of MRI at maximum growth rate is given by $L_{\mathrm{CS}} \sim 4 \sqrt{\frac{2}{3}} \frac{v_{\mathrm{A}}}{\Omega}$, where $\Omega$ is the angular velocity of the disk around the black hole, $\Omega \approx \sqrt{G M_{\mathrm{BH}} / r^{3}}>r_{\mathrm{S}} \equiv$ $2 G M_{\mathrm{BH}}$ and $v_{\mathrm{A}}$ is the Alfven velocity, $v_{\mathrm{A}}=\sqrt{B^{2} / \rho}$ ( $G$ is the gravitational constant). It shows that the magnetic Reynolds number is much larger than the unity and the resistivity is negligible for the scale of current sheet (see also page 1468, right column of Koide ${ }^{\underline{6}}$ ). With respect to Ohm's laws, we can neglect the inertia of current in all cases. Hall effect is negligible for supermassive black hole cases, while not for gamma-ray bursts (GRBs) and black hole (BH) X-ray binaries (including stellar mass black holes). On the other hand, the thermoelectromotive force would become significant compared to the resistivity in all situations. Because of the huge values of magnetic Reynolds number in Table 1, the electromotive force due to resistivity is very small compared to the ideal MHD electromotive force and then Hall effect is the same. With respect to the momentum transportation of current, it is negligible for all cases. In conclusion, we can use standard resistive RMHD equations with additional terms of thermoelectromotive force (and Hall term for GRB and BH X-ray binary.)

When we consider the pair plasma, the normalized reduced mass and normalized mass difference become $\mu=1 / 4$ and $\Delta \mu=0$ from $\mu=m_{\mathrm{e}} / m_{\mathrm{i}} \sim 1 / 1800$ and $\Delta \mu=0$ in the electron-ion plasma, respectively. Hall effect disappears and other special effects of generalized RMHD may be greater than the ratios of normal plasma by $m_{\mathrm{i}} / m_{\mathrm{e}} \sim 1800$. The conclusion with respect to the validity of the standard resistive RMHD equations is not changed.

\section{SUMMARY}

We investigated whether the standard resistive RMHD equations have the causality problem or not using the direct numerical calculations with the equations. There were two kinds of the hypothetical causality problem: (i) information transportation to the absolute past and (ii) spontaneous decrease in entropy. The numerical calculations showed that such phenomena do not appear even in cases where such phenomena are expected to happen. In the calculations, we used the electromagnetic wave packet with the characteristic length $\sigma=50$, characteristic wave number $k_{0}=2 \pi$ in the plasma with resistivity $\eta=1 /\left(2^{3 / 2} \pi\right)$. These results suggest that the "group velocity" does not have physical meaning, such as the prop- 
agation velocity of information. In fact, with respect to the first causality problem (i), we confirmed that the head velocity of the wave represents the velocity limit of the information propagation and the head velocity is the light speed in the plasma.

With respect to the second problem of causality (ii), the calculation results suggest that, in general, acausal phenomena with a wave packet never happen because the wave packet without an edge in the plasma rest frame never be transformed to the wave packet with a peak by the Lorentz transformation with the relative velocity $v_{0}>1 / v_{\mathrm{g}}$, as shown in Fig. 10 (Appendix $\mathrm{A}$ ), and the wave packet with an edge induces the (pair-soliton-like) head structure at the edge of the wave packet independently (or weakly dependent) on the bulk wave packet. The former shows that the concept of a wave packet is not consistent with the frame-invariant concept of relativity. Consequently, we understand that we have no causality problem in standard resistive RMHD as suggested by discussion with the superluminal group velocity of the electromagnetic wave ${ }^{4}$. This conclusion gives the guarantee of an usage of the standard resistive RMHD. This problem was the (mentally) biggest obstacle for application of the standard resistive RMHD equations to the global astrophysical phenomena. We expect that numerical simulations with the standard resistive RMHD equations to be more popular for analysis of plasmas around black holes, where the magnetic reconnection may play an important role in the release of the energy stored in the plasma near the black holes.

Incidentally, it is noted that in the plasma, whose plasma parameter is much larger than unity, the group velocity is less than the light speed ${ }^{\underline{4}}$. To include this effect correctly, we have to include the inertia effect of the electron or electric charge/current. That is, we have to use generalized RMHD equations to consider the high frequency phenomena, where we cannot neglect the electron or current inertia, while it is not necessary for the global, relatively slow phenomena around the astrophysical black hole.

\section{ACKNOWLEDGMENTS}

I am grateful to Mika Koide for her helpful comments on this paper. I thank Takahiro

Kudoh for the fruitful discussion. This work was supported in part by the Science Research Fund of the Japanese Ministry of Education, Culture, Sports, Science and Technology. 


\section{Appendix A: Initial condition of wave packet in a case of hypothetical acausal effect}

We show the profile of the electromagnetic wave packet propagating along the $x$ direction becomes monotonic when we observe it from the coordinates frame to the relative velocity $v_{0}>1 / v_{\mathrm{g}}$ to the rest frame of the plasma, $O-x y z$. Here, in the frame $O-x y z$, we assume that the Fourier spectrum of the wave packet is Gaussian,

$$
F(k)=\frac{\sigma}{\sqrt{2 \pi}} e^{-\frac{\sigma^{2}}{2}\left(k-k_{0}\right)^{2}}
$$

where $\sigma$ corresponds to the characteristic length of the wave packet and $k_{0}$ is the typical wave length of the wave packet. Furthermore, we assume $\sigma$ is large enough and the Fourier

spectrum is significant only near $k \sim k_{0}$. We have the complex perturbation variables of the electromagnetic wave packet as

$$
E_{y}(x, t) \propto \frac{1}{\sqrt{1+i D t / \sigma^{2}}} \exp \left[-\frac{1}{2 \sigma^{2}} \frac{\left(x-v_{\mathrm{g}} t\right)^{2}}{1+i D t / \sigma^{2}}\right] e^{-i k_{0} x-i \omega_{0} t}
$$

where $\omega_{0}=\omega\left(k_{0}\right), v_{\mathrm{g}}=(\partial \omega / \partial k)_{k=k_{0}}$, and $D=\left(\partial^{2} \omega / \partial k^{2}\right)_{k=k_{0}} \underline{\underline{4}}$. The physical solution is given by the real part of the complex perturbation, $E_{y}$. The amplitude around any point $x=x_{0}$ at any time $t=t_{0}$ is given by,

$$
\begin{aligned}
E_{y}(x, t) & \propto \frac{1}{\mid \sqrt{1+i D t / \sigma^{2}}} \exp \left[\Re\left\{-\frac{1}{2 \sigma^{2}} \frac{\left(x-v_{\mathrm{g}} t\right)^{2}}{1+i D t / \sigma^{2}}\right\}\right] e^{\left(\Im \omega_{0}\right) t}, \\
& =\frac{1}{\sqrt{1+(D t / \sigma)^{2}}} \exp \left[-\frac{1}{2 \sigma^{2}} \frac{\left(x-v_{\mathrm{g}}\right)^{2}}{1+(D t / \sigma)^{2}}\right] e^{\left(\Im \omega_{0}\right) t} .
\end{aligned}
$$

The Lorentz transform from the original coordinates frame $O-x y z$ to the new coordinates frame $O^{\prime}-x^{\prime} y^{\prime} z^{\prime}$ with the relative velocity $\boldsymbol{v}_{0}=v_{0} \hat{x}$,

$$
t^{\prime}=\gamma_{0}\left(t-v_{0} x\right), x^{\prime}=\gamma_{0}\left(x-v_{0} t\right), y^{\prime}=y, z^{\prime}=z,
$$

or

$$
t=\gamma_{0}\left(t^{\prime}+v_{0} x^{\prime}\right), x=\gamma_{0}\left(x^{\prime}+v_{0} t^{\prime}\right), y=y^{\prime}, z=z^{\prime},
$$

where $\gamma_{0}=\left(1-v_{0}^{2}\right)^{-1 / 2}$. Then, the amplitude profile of the initial electric field of the wave packet observed by the new coordinates frame $O^{\prime}-x^{\prime} y^{\prime} z^{\prime}$ is

$$
\begin{aligned}
\left|E_{y}^{\prime}\right| & =\left|\gamma_{0}\left(E_{y}-v_{0} B_{z}\right)\right|=\gamma_{0}\left|1-\frac{v_{0} k}{\omega}\right|\left|E_{y}\right| \\
& \propto \frac{1}{\left[1+\left(D \gamma_{0} v_{0} x^{\prime} / \sigma^{2}\right)^{2}\right]^{1 / 4}} \exp \left[-\frac{1}{2 \sigma^{2}} \frac{\left(1-v_{\mathrm{g}} v_{0}\right)^{2} x^{\prime 2}}{1+\left(D \gamma_{0} v_{0} x^{\prime} / \sigma^{2}\right)^{2}}\right] e^{\gamma_{0} v_{0}\left(\Im \omega_{0}\right) x^{\prime}},
\end{aligned}
$$


where we assumed $v_{0} \neq \omega_{0} / k_{0}$. Here, we have the dispersion relation of the electromagnetic wave in the plasma with the uniform resistivity $\eta$, given by Eq. (15). Then, we have

$$
\begin{aligned}
\omega_{0} & =-\frac{i}{2 \eta} \pm \frac{1}{2 \eta} \sqrt{4 \eta^{2} k^{2}-1} \\
v_{\mathrm{g}} & =\frac{2 \eta k}{\sqrt{(2 \eta k)^{2}-1}}, \\
D & =-\frac{1}{2 \eta^{2}}\left(\frac{2 \eta}{\sqrt{(2 \eta k)^{2}-1}}\right)^{3}<0 .
\end{aligned}
$$

Here, we have the relation,

$$
\frac{D}{\eta}=-\frac{1}{2}\left(\frac{v_{\mathrm{g}}}{\eta k}\right)^{3}
$$

We note that $\left|E_{y}\right|$ becomes infinite when $x^{\prime} \longrightarrow-\infty$ because $\Re \omega_{0}=-1 / \eta<0, D \neq 0$, and $v_{0} \neq 0$. This means that this wave packet keeps its finite profile only in the original frame $O-x y z$. In other coordinates frame $O^{\prime}-x^{\prime} y^{\prime} z^{\prime}$ with the finite relative velocity to the rest frame of the plasma, $O-x y z$, the electromagnetic wave can not be recognized as a wave packet anymore. Then, we understand that the concept of "wave packet" is not invariant item with respect to the Lorentz transformation.

To show the monotonicity of the initial perturbation in the new coordinates $O^{\prime}-x^{\prime} y^{\prime} z^{\prime}$, we evaluate the following variable proportional to the logarithm of the profile of the perturbation,

$$
L=2\left(\frac{D \gamma_{0} v_{0}}{\sigma}\right)^{2}\left[-\frac{\gamma_{0} v_{0}}{2 \eta} x^{\prime}-\frac{\left(1-v_{\mathrm{g}} v_{0}\right)^{2} \sigma^{2}}{2\left[\sigma^{4}+\left(D \gamma_{0} v_{0} x^{\prime}\right)^{2}\right]} x^{\prime 2}-\frac{1}{4} \log \left\{\sigma^{4}+\left(\gamma_{0} v_{0} D x^{\prime}\right)^{2}\right\}\right] .
$$

Using a new variable $\xi=-\frac{\gamma_{0} v_{0} D}{\sigma^{2}} x^{\prime}$, we have

$$
L=\frac{\left(\gamma_{0} v_{0}\right)^{2} D}{\eta} \xi+\left(v_{\mathrm{g}} v_{0}-1\right)^{2} \frac{\xi^{2}}{1+\xi^{2}}-\frac{1}{2}\left(\frac{D \gamma_{0} v_{0}}{\sigma}\right)^{2} \log \sigma^{4}\left(1+\xi^{2}\right)
$$

We evaluate the slop of $L$,

$$
\frac{\partial L}{\partial \xi}=\frac{D\left(\gamma_{0} v_{0}\right)^{2}}{\eta}\left[1-\frac{\eta D}{\sigma^{2}} \frac{\xi}{1+\xi^{2}}\right]+\left(v_{\mathrm{g}} v_{0}-1\right)^{2} \frac{2 \xi^{2}}{\left(1+\xi^{2}\right)^{2}}
$$

When $\xi$ is greater than zero, it is clear that $\partial L / \partial \xi$ is negative. When $\xi$ is negative, $\partial L / \partial \xi$ is negative if

$$
\frac{1}{\gamma_{0}^{2}} \frac{\partial L}{\partial \xi}<-4 v_{0}^{2}\left(v_{\mathrm{g}}^{2}-1\right)^{3 / 2}+\left(\frac{\sqrt{3}}{2}\right)^{3}\left(v_{\mathrm{g}} v_{0}-1\right)^{2}\left(1-v_{0}^{2}\right)+2 v_{0}^{2}\left(v_{\mathrm{g}}^{2}-1\right)^{3} \frac{4 \eta^{2}}{\sigma^{2}}<0
$$


where we use inequalities, $\left|2 \xi /\left(1+\xi^{2}\right)\right| \leq 1$ and $\left|2 \xi /\left(1+\xi^{2}\right)^{2}\right| \leq(\sqrt{3} / 2)^{3}$. When $v_{0}>1 / v_{\mathrm{g}}$ and $v_{\mathrm{g}}>1$, we have

$$
\left(v_{\mathrm{g}} v_{0}-1\right)^{2}\left(1-\frac{1}{v_{0}^{2}}\right)<\left(v_{\mathrm{g}}^{2 / 3}-1\right)^{3}
$$

and

$$
\frac{\left(v_{\mathrm{g}}^{2 / 3}-1\right)^{3}}{\left(v_{\mathrm{g}}^{2}-1\right)^{3 / 2}}<(2 \cos 4 \pi / 9)^{3 / 2} \frac{(1-2 \cos 4 \pi / 9)^{3}}{\left(1-8 \cos ^{3} 4 \pi / 9\right)^{3 / 2}},
$$

where $2 \cos 4 \pi / 9=0.3472 \cdots$. Then, $\partial L / \partial \xi$ is negative if

$$
\begin{aligned}
& \frac{1}{\gamma_{0}^{2}} \frac{\partial L}{\partial \xi}<2 v_{0}\left(v_{0}^{2}-1\right)^{3}\left[-\frac{2}{\left(v_{\mathrm{g}}^{2}-1\right)^{3 / 2}}\left\{1-\frac{1}{4}\left(\frac{\sqrt{3}}{2}\right)^{3} \times 0.35^{3 / 2}(1-1 / 3)^{3}\right\}+\frac{4 \eta^{2}}{\sigma^{2}}\right] \\
& <2 v_{0}\left(v_{0}^{2}-1\right)^{3}\left[-\frac{2}{\left(v_{\mathrm{g}}^{2}-1\right)^{3 / 2}} \times 0.989993+\frac{4 \eta^{2}}{\sigma^{2}}\right]<0 .
\end{aligned}
$$

On the other hand, because we neglect the third order terms of $\left|k-k_{0}\right| \leq 1 / \sigma$ with respect to $\omega$, we already assumed that

$$
\left|\frac{\frac{1}{3 !} \frac{\partial^{3} \omega}{\partial k^{3}} \frac{1}{\sigma^{3}}}{\frac{1}{2 !} \frac{\partial^{2} \omega}{\partial k^{2}} \frac{1}{\sigma^{2}}}\right|=\frac{1}{3}\left|\frac{1}{D} \frac{\partial D}{\partial k}\right| \frac{1}{\sigma} \ll 1,
$$

where $D=\partial^{2} \omega / \partial k^{2}=-4 \eta /\left[(2 \eta k)^{2}-1\right]^{3 / 2}$. When we use the relation $\left((2 \eta k)^{2}-1\right)^{-1}=v_{\mathrm{g}}^{2}-1$, we have

$$
\frac{4 \eta k\left(v_{\mathrm{g}}^{2}-1\right) \eta}{\sigma} \ll 1
$$

Using the relation, $\frac{v_{\mathrm{g}}}{\eta k}=2\left(v_{\mathrm{g}}^{2}-1\right)^{1 / 2}$, we have

$$
\frac{\eta}{\sigma} \ll \frac{1}{2 v_{\mathrm{g}}\left(v_{\mathrm{g}}^{2}-1\right)^{1 / 2}}
$$

and then we obtain

$$
\frac{4 \eta^{2}}{\sigma^{2}} \ll \frac{4}{2 v_{\mathrm{g}}^{2}\left(v_{\mathrm{g}}^{2}-1\right)}<\frac{2}{v_{\mathrm{g}}^{3}\left(1-1 / v_{\mathrm{g}}^{2}\right)^{3 / 2}}<\frac{2}{\left(v_{\mathrm{g}}^{2}-1\right)^{3 / 2}} .
$$

Then, we find

$$
\frac{4 \eta^{2}}{\sigma^{2}}<\frac{2}{\left(v_{\mathrm{g}}^{2}-1\right)^{3 / 2}} \times 0.989993 .
$$

This confirms $\partial L / \partial \xi$ and then $\partial L / \partial x^{\prime}$ are always negative. This calculation shows that the slope of the transformed shape of the original wave packet into the new coordinates with $v_{0}>1 / v_{\mathrm{g}}$ is monotonically decreasing function, and its shape is drastically different from that set in the plasma rest frame. That is, the concept of "wave packet" with infinite length is not invariant for the Lorentz transformation, and contradicts with relativity. 


\section{Appendix B: Lorentz invariant property of head velocity}

We show the frame transport property of the head velocity of a wave train. We consider two different inertial frames $x^{\mu}$ and $x^{\mu \prime}$, where the metric is given by

$$
d s^{2}=\eta_{\mu \nu} d x^{\mu} d x^{\nu}=\eta_{\rho \sigma} d x^{\rho \prime} d x^{\sigma \prime}
$$

Then, we have the relation with respect to the transportation coefficient between the two frames,

$$
\eta_{\mu \nu}=\eta_{\rho \sigma} \frac{\partial x^{\rho \prime}}{\partial x^{\mu}} \frac{\partial x^{\sigma \prime}}{\partial x^{\nu}}=-\frac{\partial x^{0 \prime}}{\partial x^{\mu}} \frac{\partial x^{0 \prime}}{\partial x^{\nu}}+\sum_{i} \frac{\partial x^{i \prime}}{\partial x^{\mu}} \frac{\partial x^{i \prime}}{\partial x^{\nu}} .
$$

The transform of the 4 -wavenumber $k^{\mu}=(\omega, \boldsymbol{k})$ is given by

$$
\begin{aligned}
\omega^{\prime} & =\frac{\partial x^{0 \prime}}{\partial x^{0}} \omega+\sum_{i} \frac{\partial x^{0 \prime}}{\partial x^{i}} k^{i}, \\
k^{i \prime} & =\frac{\partial x^{i \prime}}{\partial x^{0}} \omega+\sum_{j} \frac{\partial x^{i \prime}}{\partial x^{j}} k^{j} .
\end{aligned}
$$

The wave head velocity in the coordinates frame $O^{\prime}-x^{\prime} y^{\prime} z^{\prime}$ is calculated

$$
\begin{aligned}
v_{\mathrm{h}}^{\prime} & =\lim _{\omega^{\prime} \longrightarrow \infty} \frac{\omega^{\prime}}{k^{\prime}}=\lim _{\omega^{\prime} \longrightarrow \infty} \frac{\omega^{\prime}}{\sqrt{\sum_{i} k_{i}^{\prime 2}}} \\
& =\lim _{\omega^{\prime} \longrightarrow \infty} \frac{\frac{\partial x^{0 \prime}}{\partial x^{0}} \omega+\sum_{i} \frac{\partial x^{0 \prime}}{\partial x^{i}} k^{i}}{\left[\sum_{i}\left(\frac{\partial x^{i \prime}}{\partial x^{0}} \omega+\sum_{j} \frac{\partial x^{i \prime}}{\partial x^{j}} k^{j}\right)^{2}\right]^{1 / 2}} \\
& =\frac{\frac{\partial x^{0 \prime}}{\partial x^{0}} v_{\mathrm{h}}+\sum_{i} \frac{\partial x^{0 \prime}}{\partial x^{i}} n^{i}}{\left[\sum_{i}\left(\frac{\partial x^{i \prime}}{\partial x^{0}} v_{\mathrm{h}}+\sum_{j} \frac{\partial x^{i \prime}}{\partial x^{j}} n^{j}\right)^{2}\right]^{1 / 2}},
\end{aligned}
$$

where we define $n^{i}=k^{i} / k$. Using Eq. (B21), we derive

$$
\sum_{i}\left(\frac{\partial x^{i \prime}}{\partial x^{0}} v_{\mathrm{h}}+\sum_{j} \frac{\partial x^{i \prime}}{\partial x^{j}} n^{j}\right)^{2}=\left(\frac{\partial x^{0 \prime}}{\partial x^{0}} v_{\mathrm{h}}+\sum_{j} \frac{\partial x^{0 \prime}}{\partial x^{j}} n^{j}\right)^{2}+1-v_{\mathrm{h}}^{2}
$$

Then, if $v_{\mathrm{h}} \leq 1$, we have $v_{\mathrm{h}}^{\prime} \leq 1$. The equality stands only if $v_{\mathrm{h}}=1$ in a certain frame.

\section{REFERENCES}

${ }^{1}$ S. Koide, T. Kudoh, \& K. Shibata, Physical Review D, 74, 044005 (2006).

${ }^{2}$ J. C. McKinney, Monthly Notice of Royal Astronomical Society, 368, 1561 (2006). 

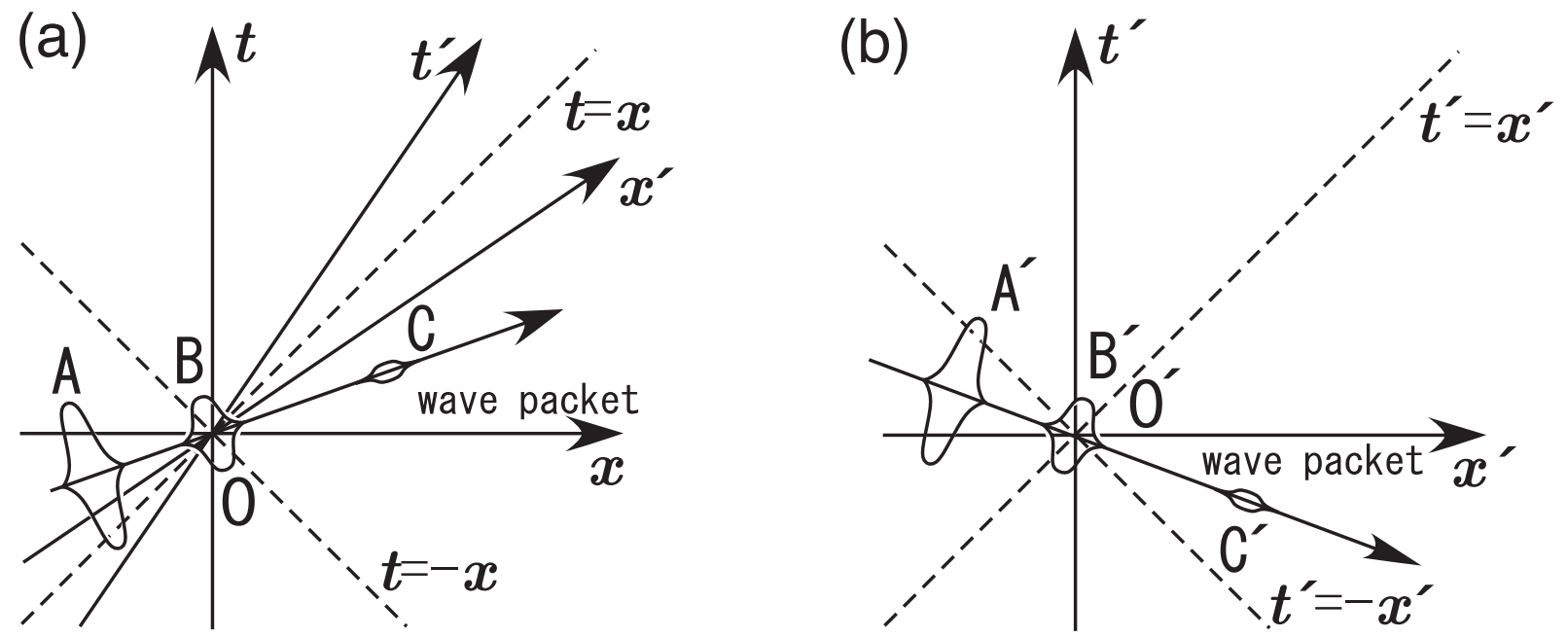

FIG. 1. Propagation of superluminal electromagnetic wave packet in uniform, unmagnetized plasma. (a) In the rest plasma coordinates frame $O-x y z$, the wave packet propagates and damps as shown by the profiles at the points $\mathrm{A}, \mathrm{B}$, and $\mathrm{C}$. (b) In the coordinates frame with the relative velocity to the rest frame, $v_{0}>1 / v_{\mathrm{g}},\left(O^{\prime}-x^{\prime} y^{\prime} z^{\prime}\right) \mathrm{A}, \mathrm{B}$, and $\mathrm{C}$ in the panel (a) correspond to the points A', B', and C', respectively. In this frame $O^{\prime}-x^{\prime} y^{\prime} z^{\prime}$, the wave packet seems to propagate opposite direction of the wave in the plasma rest frame and grow up.

${ }^{3}$ J. D, Jackson, Classical Electrodynamics, (John Wiley \& Sons, New York, 1972).

${ }^{4}$ S. Koide, Physical Review D, 78, 125026 (2008).

${ }^{5}$ S. Koide, Astrophysical Journal, 696, 2220 (2009).

${ }^{6}$ S. Koide, Astrophysical Journal, 708, 1459 (2010).

${ }^{7}$ S. Weinberg, Gravitation and Cosmology (John Wiley \& Sons, New York, 1972).

${ }^{8}$ N. Watanabe, \& T. Yokoyama, Astrophysical Journal, 647, L123 (2006).

${ }^{9}$ K. Miyamoto, Plasma Physics for Nuclear Fusion (MIT Press, Cambridge, 1989).

${ }^{10}$ J. F. Hawley \& S. A. Balbus, Astrophysical Journal, 400, 595 (1992).

${ }^{11}$ T. Sano \& S.-I. Inutsuka, Astrophysical Journal, 561, L179 (2001).

${ }^{12}$ J. C. McKinney, PhD thesis, Univ. Illinois (2004). 
Group velocity and causality in standard resistive RMHD
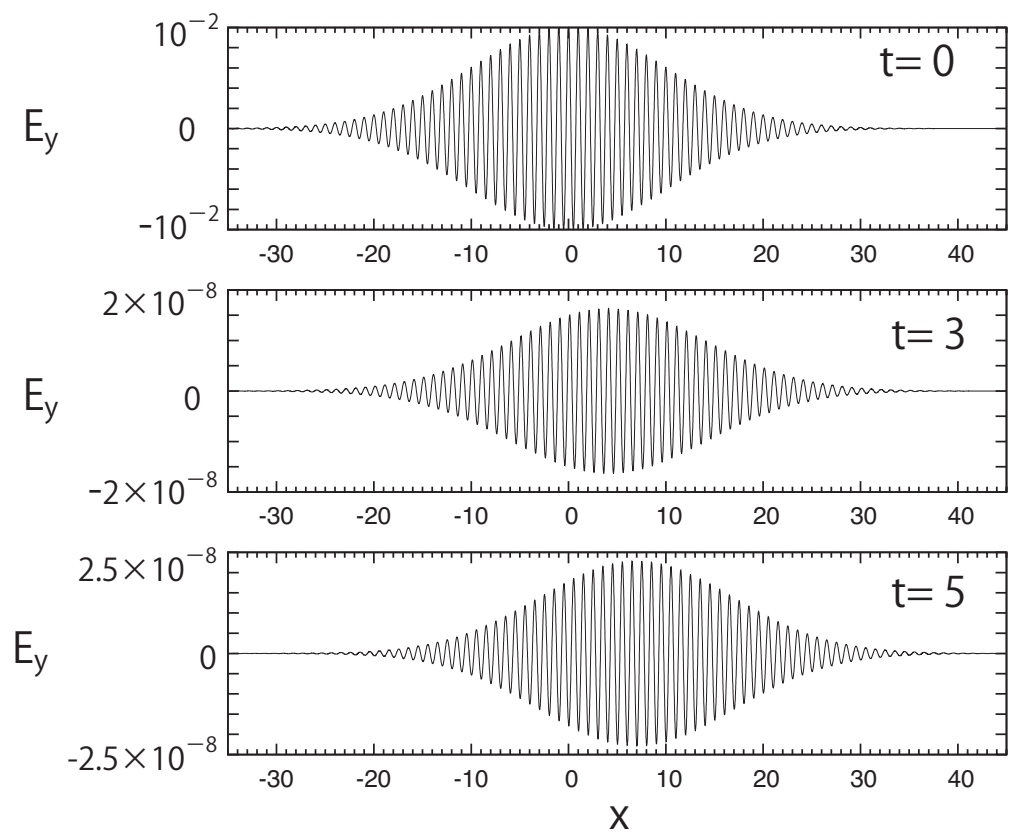

FIG. 2. Simulation of propagation of a simple wave packet with a narrow Fourier spectrum.
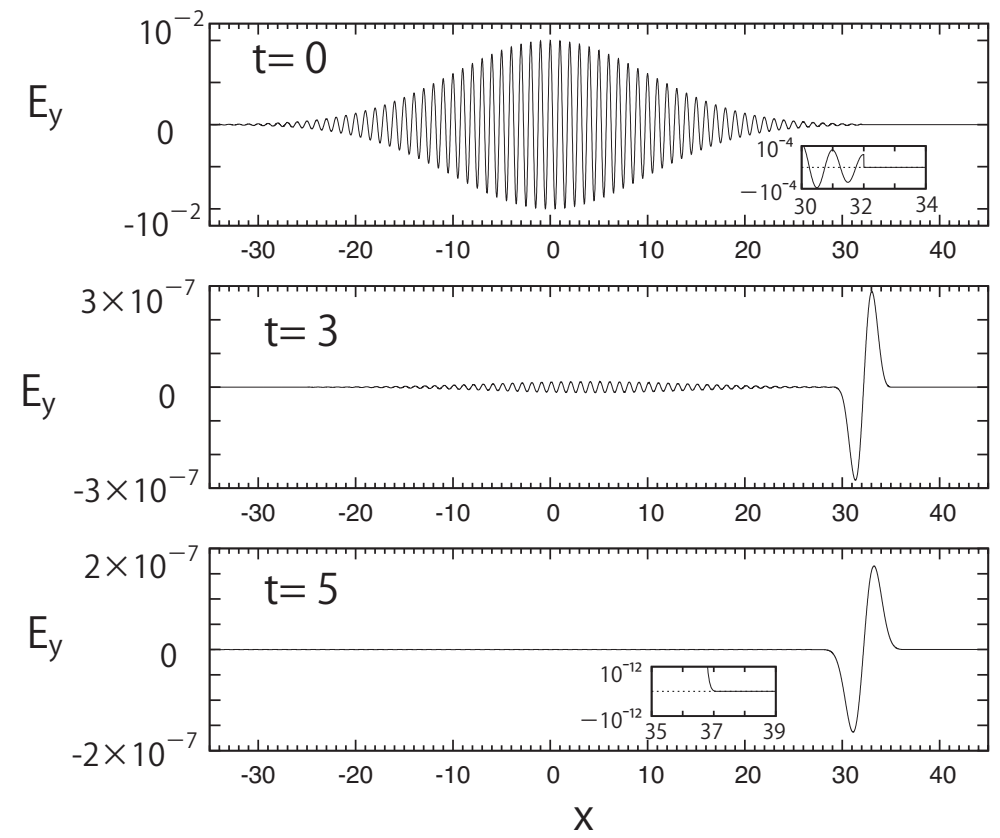

FIG. 3. Simulation of a wave packet with a head edge. The small boxes in the top and bottom panels show the zoom-up of the profiles of the head edge of the wave packet in the range $30 \leq x \leq$ $34,-10^{-4} \leq E_{y} \leq 10^{-4}$ and $35 \leq x \leq 39,-10^{-12} \leq E_{y} \leq 10^{-12}$ at $t=0$ and $t=5$, respectively. The latter small panel shows that the head edge locates at $x=37$ at $t=5$. 
Group velocity and causality in standard resistive RMHD
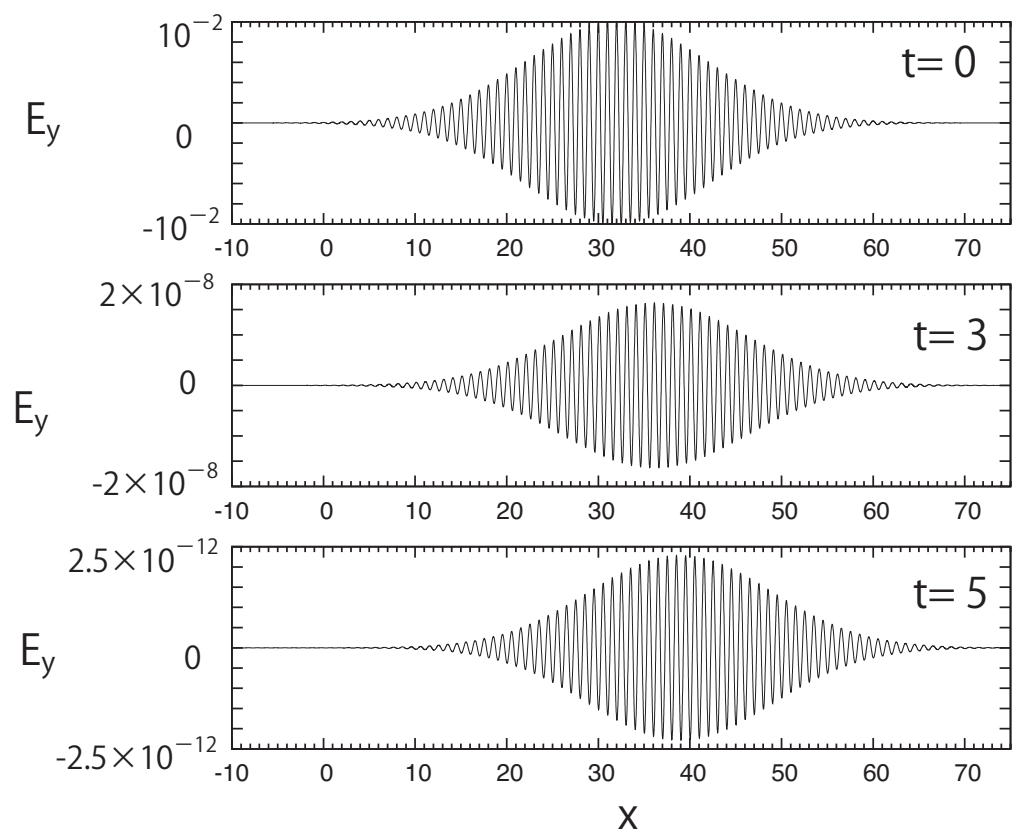

FIG. 4. Simulation of propagation of wave packet with a very narrow Gaussian Fourier spectrum. The initial condition of this wave packet shifts toward the right direction by $\Delta x=32$ from the calculation of Fig. 1. 
Group velocity and causality in standard resistive RMHD

(a)
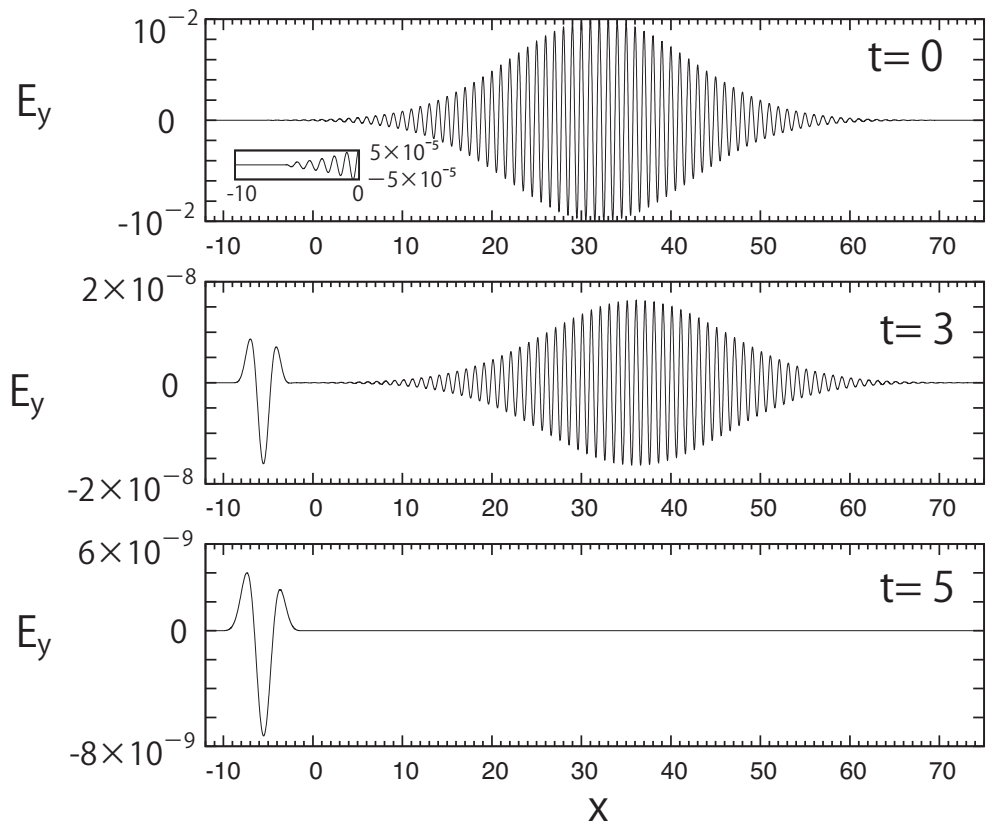

(b)
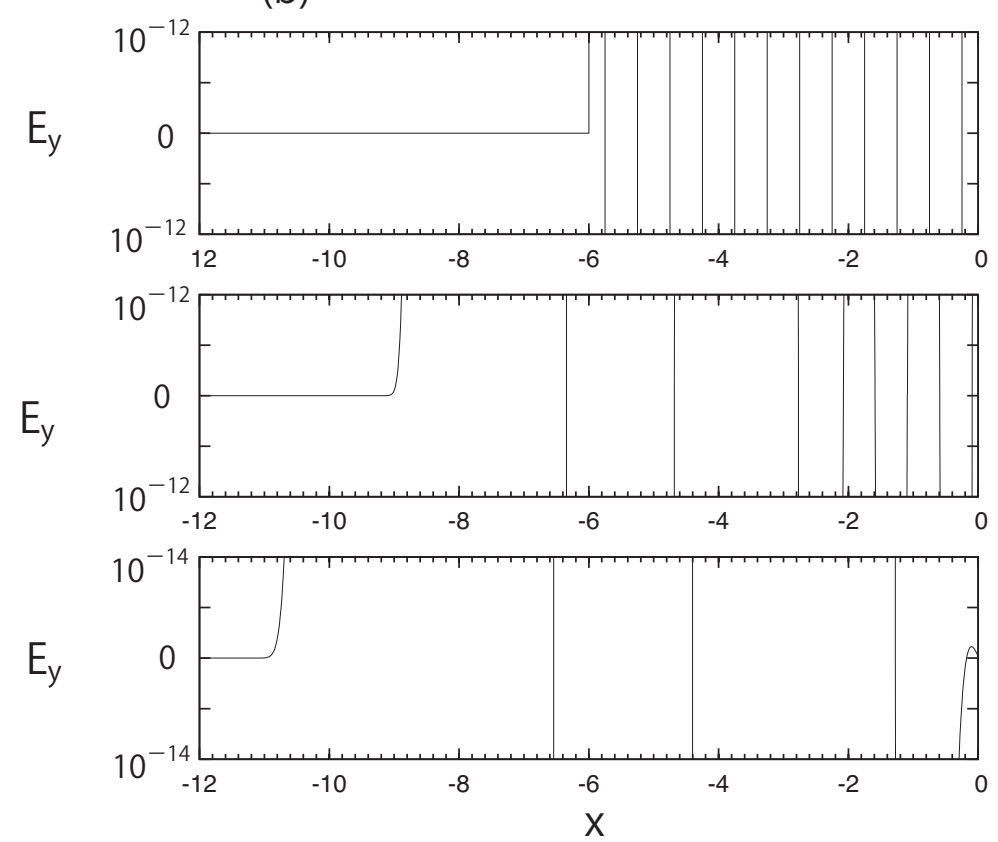

FIG. 5.

Simulation of propagation of a wave packet with a back edge in the plasma rest frame $O-x y z$.

(a) Whole profiles of the wave packet. (b) Profiles of the wave packet around the rear edge. The small box in the top panel shows the rear edge of the wave packet in the range $-10 \leq x \leq 0$,

$$
-5 \times 10^{-5} \leq E_{y} \leq 5 \times 10^{-5} .
$$


Group velocity and causality in standard resistive RMHD
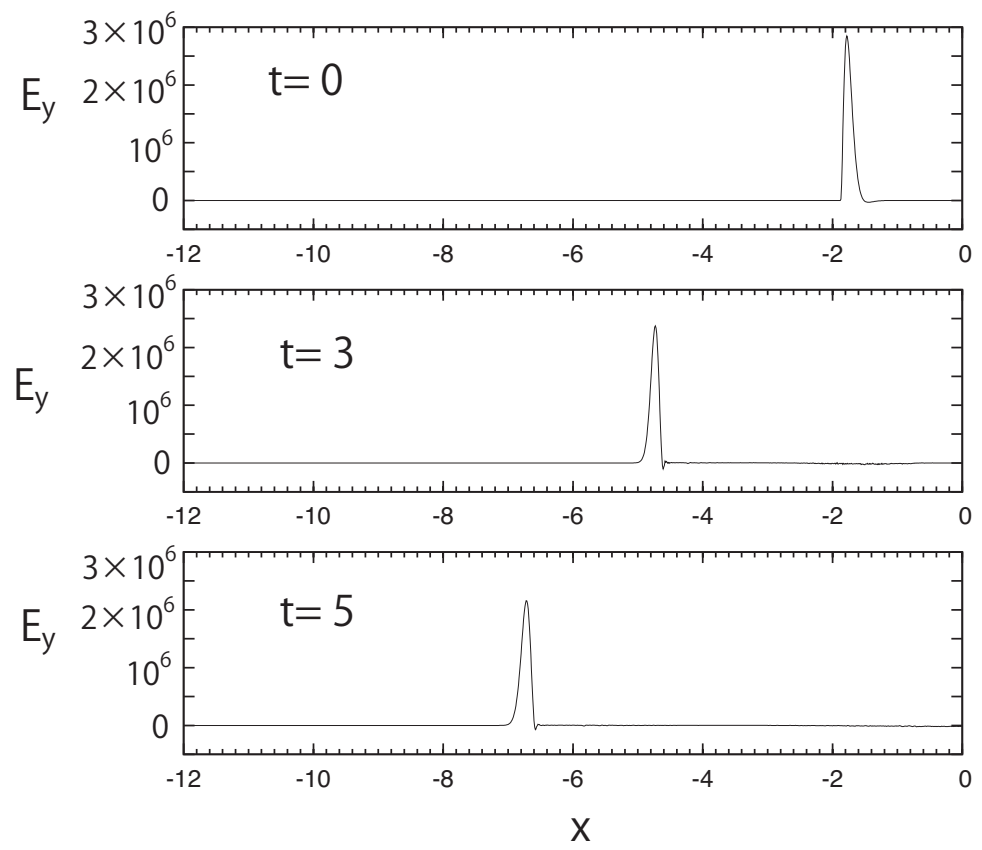

FIG. 6. Simulation of propagation of a wave packet in the coordinates frame where the plasma flows toward the left direction with $v_{0}>1 / v_{\mathrm{g}}$. This coordinates frame corresponds to the new frame $O^{\prime}-x^{\prime} y^{\prime} z^{\prime}$.

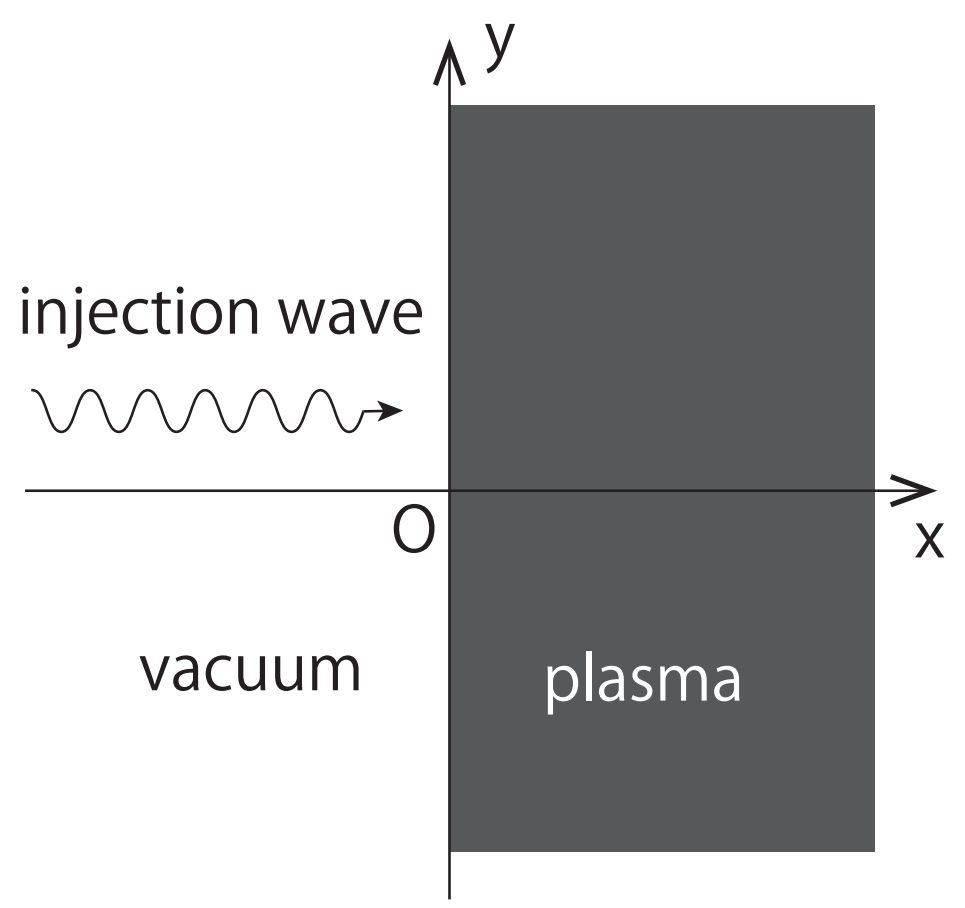

FIG. 7. Injection of electromagnetic simple plane wave from the vacuum to the semi-infinite uniform plasma at $x \geq 0$. 


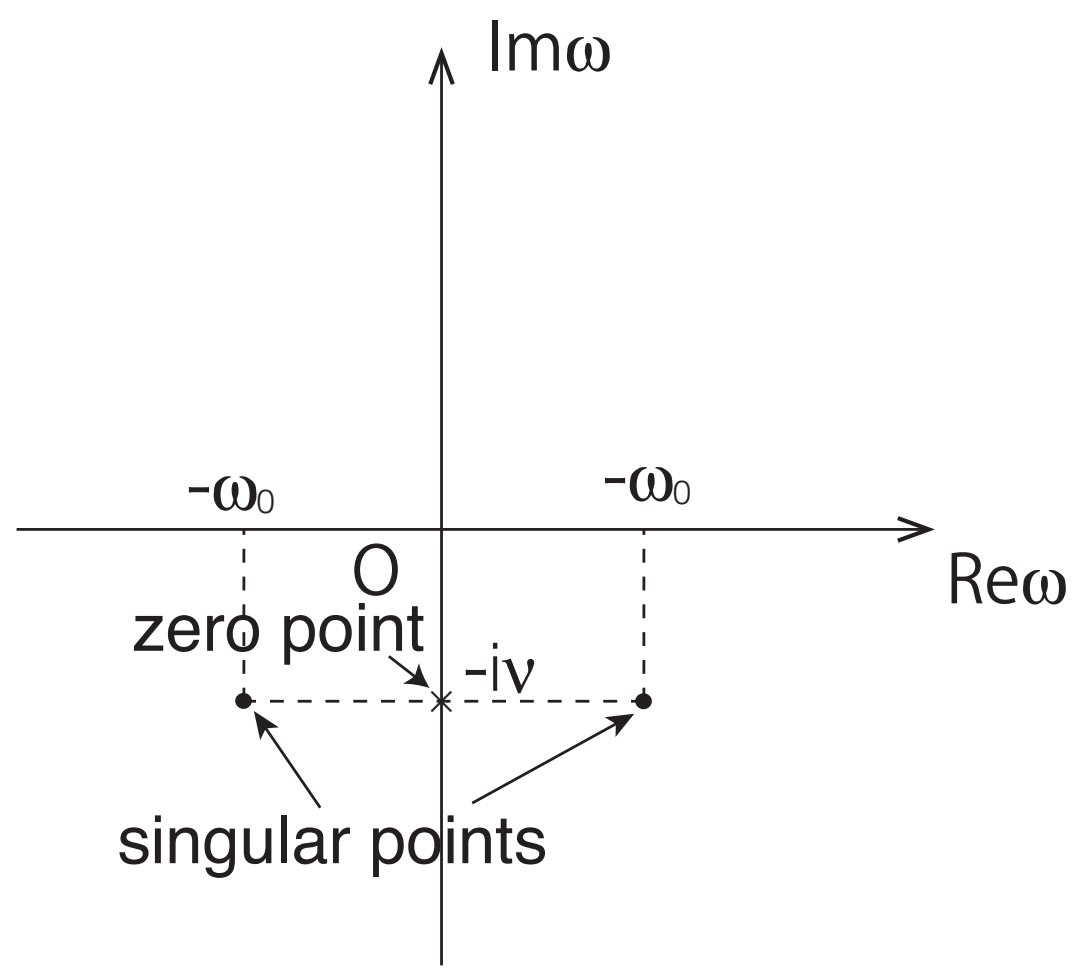

FIG. 8. Singular and zero points of $A(\omega)$ on the $\omega$ complex plane.

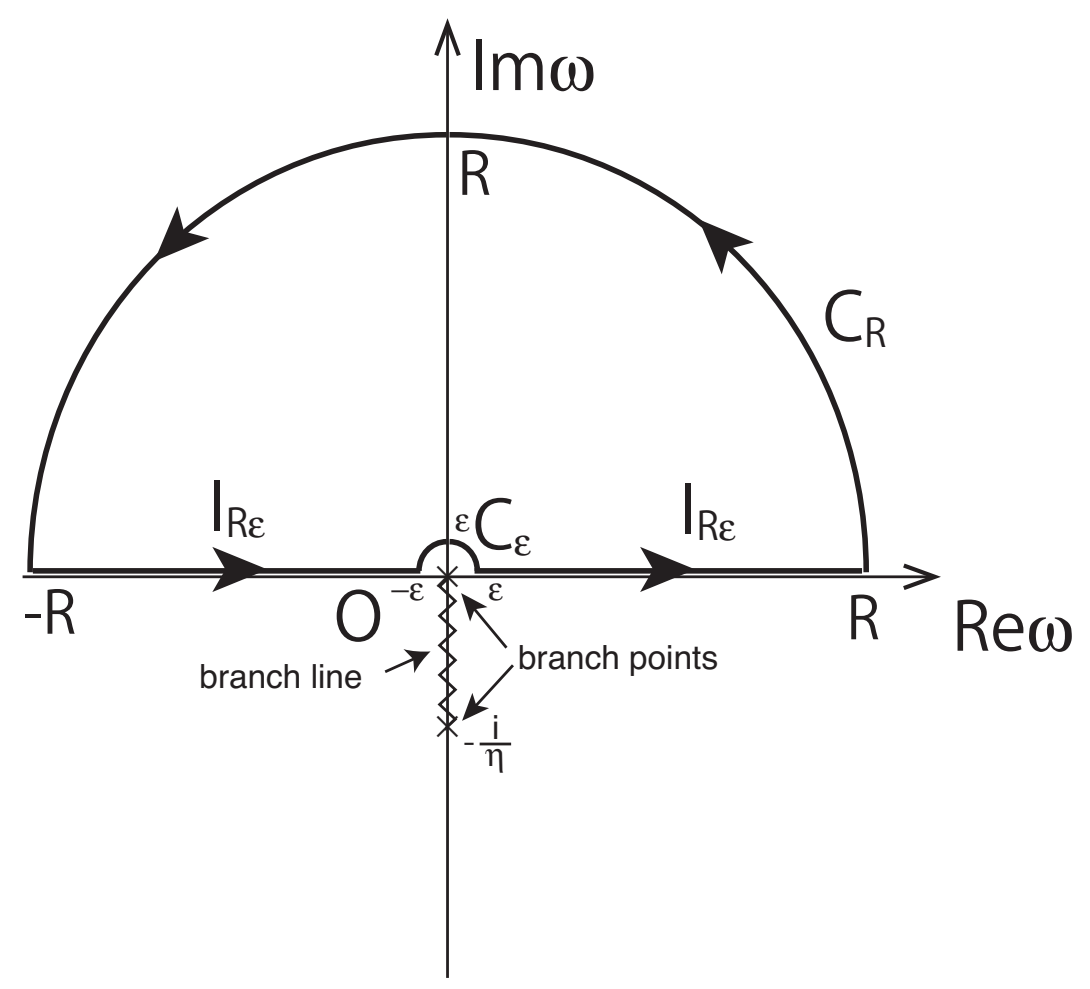

FIG. 9. Branch points and a branch line of $k(\omega)$ on the complex $\omega$ plane and the path of the contour integral for the amplitude of the electric field (Eq. (24)). 
Group velocity and causality in standard resistive RMHD

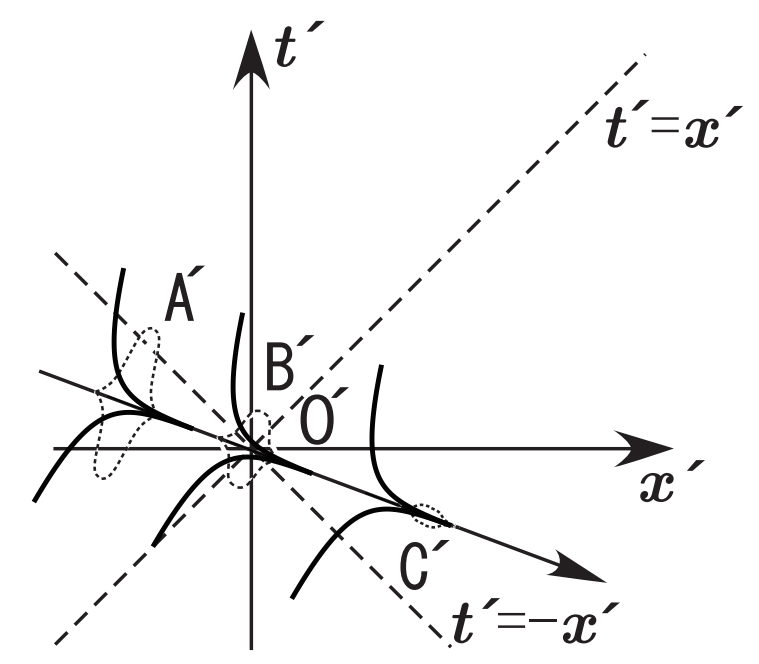

FIG. 10. Corrected image of the panel (b) of Fig. 1, In the frame $O^{\prime}-x^{\prime} y^{\prime} z^{\prime}$, the profile of the electromagnetic wave becomes monotonic and transports no signal because it has no peak. 
Group velocity and causality in standard resistive RMHD

TABLE I. Microscopic variables $\left(\omega_{\mathrm{p}}^{\mathrm{e}-\mathrm{i}}, \omega_{\mathrm{c}}^{\mathrm{e}-\mathrm{i}}, \cdots\right)$ and characteristic scales of phenomena of plasmas around black holes to examine validity of the standard resistive RMHD equations.

\begin{tabular}{|c|c|c|c|c|}
\hline & GRB & BH X-ray binary & $\begin{array}{c}\text { Supermassive BH } \\
\text { in Galaxy }\end{array}$ & AGN \\
\hline Objects & GRB030329 & LMC X-3 & Sgr A* & M87 \\
\hline$M_{\mathrm{BH}}\left[M_{\odot}\right]^{\mathrm{a}}$ & 3 & 10 & $2.6 \times 10^{6}$ & $3 \times 10^{9}$ \\
\hline$\dot{M}_{-}^{\mathrm{a}}$ & $0.1 M_{\odot} \mathrm{s}^{-1}$ & $10^{-8} M_{\odot} \mathrm{yr}^{-1}$ & $10^{-5} M_{\odot} \mathrm{yr}^{-1}$ & $10^{-2} M_{\odot} \mathrm{yr}^{-1}$ \\
\hline$\rho\left[\mathrm{g} \mathrm{cm}^{-3}\right\rfloor^{\mathrm{a}}$ & $1.6 \times 10^{10}$ & 0.0072 & $1.5 \times 10^{-6}$ & $1.3 \times 10^{-8}$ \\
\hline$T_{\mathrm{e}}[\mathrm{K}]^{\mathrm{a}}$ & $1.2 \times 10^{10}$ & $1.8 \times 10^{8}$ & $3.5 \times 10^{5}$ & $7.1 \times 10^{4}$ \\
\hline$B[\mathrm{G}]^{\mathrm{a}}$ & $2.7 \times 10^{14}$ & $1.1 \times 10^{6}$ & $1.4 \times 10^{2}$ & 3.7 \\
\hline$n_{\mathrm{e}}\left[\mathrm{m}^{-3}\right]$ & $0.9 \times 10^{39}$ & $4 \times 10^{27}$ & $0.9 \times 10^{24}$ & $0.8 \times 10^{22}$ \\
\hline$T_{\mathrm{e}}[\mathrm{eV}]$ & $1.2 \times 10^{6}$ & $2 \times 10^{4}$ & $3.5 \times 10$ & 7 \\
\hline$\nu_{\mathrm{ei}}\left[\mathrm{s}^{-1}\right]$ & $4.3 \times 10^{19}$ & $0.9 \times 10^{11}$ & $3 \times 10^{11}$ & $3 \times 10^{10}$ \\
\hline$S_{\mathrm{M}}=\frac{\mu \omega_{\mathrm{p}}^{2}}{\omega \nu_{\mathrm{ei}}}$ & $5 \times 10^{14}$ & $2 \times 10^{9}$ & $3 \times 10^{8}$ & $3 \times 10^{9}$ \\
\hline$\omega_{\mathrm{p}}^{\mathrm{e}-\mathrm{i}}\left[\mathrm{s}^{-1}\right]$ & $5.4 \times 10^{21}$ & $3.6 \times 10^{15}$ & $5.3 \times 10^{13}$ & $4.9 \times 10^{12}$ \\
\hline$\omega_{\mathrm{c}}^{\mathrm{e}-\mathrm{i}}\left[\mathrm{s}^{-1}\right]$ & $2.5 \times 10^{18}$ & $1.0 \times 10^{10}$ & $1.3 \times 10^{6}$ & $3.5 \times 10^{4}$ \\
\hline$L<L_{\mathrm{CS}}[\mathrm{m}]^{\mathrm{b}}$ & 480 & 10 & $2.3 \times 10^{4}$ & $7.5 \times 10^{5}$ \\
\hline$\tau=\omega^{-1}<L_{\mathrm{CS}} / c[\mathrm{~s}]^{\mathrm{b}}$ & $1.6 \times 10^{-6}$ & $3.3 \times 10^{-8}$ & $7.7 \times 10^{-5}$ & $2.5 \times 10^{-3}$ \\
\hline inertia of current, $\mu \frac{\omega}{\nu_{\mathrm{ei}}}$ & $8 \times 10^{-18}$ & $2 \times 10^{-7}$ & $2 \times 10^{-11}$ & $7 \times 10^{-12}$ \\
\hline $\begin{array}{l}\text { Hall effect, } \Delta \mu \frac{\omega_{\mathrm{c}}}{\nu_{\mathrm{ei}}} \\
\text { thermoelectromotive }\end{array}$ & 0.06 & 0.1 & $4 \times 10^{-6}$ & $1 \times 10^{-6}$ \\
\hline $\begin{array}{l}\text { force, } \leq \frac{\mu \omega_{\mathrm{p}}^{2}}{\nu_{\mathrm{e}} \omega_{\mathrm{c}}} \\
\text { momentum of charge } \\
\frac{2 \mu \omega_{\mathrm{c}}}{S_{\mathrm{M}} \nu_{\mathrm{ei}}}\end{array}$ & $1 \times 10^{-19}$ & $6 \times 10^{-14}$ & $2 \times 10^{-17}$ & $4 \times 10^{-19}$ \\
\hline
\end{tabular}

${ }^{a}$ Data are from McKinney 12

b The minimum values of $L$ and $\tau$ are estimated by the thickness $\left(L_{\mathrm{CS}}\right)$ and the light transit time $\left(L_{\mathrm{CS}} / c\right)$ of the current sheet caused by magneto-rotational instability (MRI) in the disk. 